[3] E. Dyer and II. E. Hamstrom, Completely regular mappings, Fund. Math 45 (1958), pp. 113-118.

B. Nadler, The dimension of certain hyperspaces, Bull. Polon. Acad. Sci. Sér. Sci. Math. Astronom. Phys. 19 (1971), pp. 1027-1034.

] W. Henderson, On the hyperspaces of subcontinua of an arc-like continuum,

[5] G. W. Henct 27 (1971), pp. 416-417.

J. L. Kelley, Hyperspaces of a continuum, Trans. Amer. Math. Soc. 52 (1942), pp. $22-36$.

[7] J. Krasinkiewicz, No 0-dimensional set disconnects the hyperspace of a continuum Bull. Polon. Acad. Sci. Sér. Sci. Math. Astronom. Phys. 19 (1971), pp. 755-758. Bull the hyperspaces of snake-like and circle-lite continua (to appear).

[8] - On the hyperspaces of snake-like 2, New York-London-Warszawa 1968.

[9] K. Kuratowski, Topology, vol. 2, New York-London-Warszaces (to appear).

[10] S. B. Nadler, Jr., Locating cones and Hilbert oubes in hyperspaces (to appear).

[11] T. Nishiura and C. S. Ri (1972), pp. 550-556.

Pr. Amer.

12] C. J. Rhee, Onences de Liege, 38 Année, 11-12 (1969), pp. 602-604.

13] J. T. Rogers, The cone=hyperspace property, Can. J. Math. 24 (1972), pp. 279-285.

14] - Embedding the hyperspaces of circle-like plane continua, Proc. Amer. Math. Soc. 29 (1971), pp. 165-168.

[15] W. R. R. Transue, On the hyperspace of subcontinua of the pseudoarc, Proc. Amer. Math. Soc. 18 (1967), pp. 1074-1075.

[16] H. Whitney, Regular families of curves, Proc. Nat. Acad. Sci., U.S.A. 18 (1932), pp. 275-288.

[17] G.S. Young, The introduction of local connectivity by change of topology, Amer. J. Math. 68 (1946), pp. 479-494.

\title{
Classes of Dedekind finite cardinals $\left(^{(1)}\right.$
}

by

\author{
John Truss (Oxford)
}

Abstract. We discuss seven possible definitions of "finiteness" of cardinal numbers, and associate with each definition the class of cardinals "finite" in that sense. The results are extensions of those in Levy's paper "The independence of various definitions of finiten" (Fund "Math. 46 (1958), pp. 1-13). We investigate the closure of the classes under addition, multiplication, unions, and disjoint unions. In the final section we give under addition, multiplication, unions, and dinclusions and equalities between the classes. a wide variety of possible combinations of inclusions and equalities between the classes.
Also we give an affirmative answer to Tarski's. question "Can there be exactly $2^{* 0} \mathrm{De}$ dekind finite cardinals?"

\$1. The object of this paper is to investigate various properties of classes of Dedekind finite cardinals, and to discover what possible combinations of inclusions and equalities can hold between them. The starting point is Levy's paper "The independence of various definitions of finiteness" [8]. All but two of the seven classes correspond to definitions of Levy (which were in turn taken from Tarski [18]), and the other two arise quite naturally.

The classes are defined in $\S 2$, and some of their elementary properties given. In $\S 3$ we discuss the closure of the classes under,$+ \times$, unions, disjoint mions, and $\leqslant$, and a model is given in which $\Delta_{5}$ is not closed under $x$. In $\S 4$ we show that if any two Dedekind finite cardinals are comparable, then any infinite set is the disjoint union of two infinite sets. $\$ 5$ contains five models which establish various possibilities of strict inclusion and equality between the classes. Various combinations of these models actually yield thirteen models in which the combination of strict inclusion and equality is different. It is shown that there can be no more than twenty-three possible combinations, using the results of $\$ 2$ and $\& 3$. All of the ten unsolved cases involve the following situation:

There is an infinite set with no infinite orderable subset, and if a set $X$ has a countable partition, there is a map from $X$ onto $X$ which is not $1-1$.

(1) This paper is part of the author's $\mathrm{Ph}$. D. thesis at the University of Leeds. He would like to thank Dr. F. R. Drake and Prof. A. Levy for their supervision, and the Science Research Council for their financial support. 
We do not know if this is possible, and suspect that it is.

One or two results are proved incidentally which are of interest apart from the immediate setting. One of them has been mentioned above, namely that if any two Dedekind finite cardinals are comparable, then $\omega=\dot{A}_{1}$ (in the notation of $\S 2$ ). This is a very small start to answering Tarski's question as to whether the comparability of any two members of $\Delta$ implies that $\omega=\Delta$. Another incidental result is a strengthening of Läuchli's result [6] that

W: any set of non-empty well-orderable sets has a choice function, does not imply the ordering principle.

We show that $W$ does not even imply that any infinite set has an infinite ordered partition, and at the same time avoid any group-theoretical complications. (The model is due to Gauntt [3].)

Finally we show that in Halpern-Levy's model [4] (our $\mathfrak{R}_{1}$ of Theorem 5), there are exactly $2^{N_{0}}$ Dedekind finite cardinals, thus answering affirmatively another question of Tarski ${ }^{2}$ ). (see [17]). We also show that $\mathfrak{N}_{2}$ and $\mathfrak{N}_{5}$ of Theorem 6 (which are Fraenkel-Mostowski models) satisfy $|\Delta|={ }_{0} 2^{N_{0}}$, and hence $\Delta$ can be well-ordered. In fact it follows easily that $\mathfrak{R}_{\text {, and }} \mathfrak{N}_{5}$ satisfy "any set of cardinals can be well-ordered".

Most of our notation is standard. If $x$ and $y$ are cardinals, we write $x \leqslant^{*} y$ to mean that whenever $|X|=x$ and $|Y|=y$, there is a mapping from a subset of $Y$ onto $X$. A class $Q$ of cardinals is closed under unions if whenever $|X| \epsilon Q$, and $\xi \in X \rightarrow|\xi| \epsilon Q$, then $|\bigcup X| \epsilon Q . Q$ is closed under disjoint unions if whenever $X$ is disjointed, $|X| \in Q$, and $\xi \in Q \rightarrow|\xi| \epsilon Q$, then $\| X \mid \in Q . Q$ is 'closed under $\times$ (+ respectively) if whenever $x, y \in Q$, then $x y \in Q(x+y$ respectively), and $Q$ is closed under $\leqslant$ if whenever $x \in Q, y \leqslant x$, then $y \in Q$.

§ 2. The seven classes of Dedekind finite cardinals are as follows.

$$
\omega=\{n: n \text { a finite ordinal }\} \text {. }
$$

(From now on "finite" means "has cardinal in $\omega "$.)

$$
\begin{aligned}
& \Delta_{1}=\{x: x=y+z \rightarrow y \text { or } z \text { finite }\}, \\
& \Delta_{2}=\{|X|: \text { any ordered partition of } X \text { is finite }\} \\
& \Delta_{3}=\{|X|: \text { any ordered subset of } X \text { is finite }\}, \\
& \Delta_{4}=\left\{x: \wedge_{0} \star^{*} x\right\}, \\
& \Delta_{5}=\left\{x: x+1 \star^{*} x\right\}, \\
& \Delta=\left\{x: \wedge_{0} \nless x\right\} .
\end{aligned}
$$

(2) This question was first brought to our attention by Dr J.E. Rubin. See her forthcoming joint paper with A. L. Rubin: "The cardinality of the set of Dedekind finite cardinals in Fraenkel-Mostowski models".
Of these, $\Delta_{3}$ and $\Delta_{5}$ are new, so far as we know, and the others appear in Le'vy [8]. The significance of $\Delta_{5}$ is that it comprises all cardinals which can be cancelled additively from $\leqslant *$-inequalities. $\Delta_{3}$ is considered because of the obvious parallel with $\Delta_{2}$, and that $\Delta_{2}$ comprises just those cardinals. finite in sense II of Levy in shown now.

LEMIMA 1. $|X| \in \Delta_{2}$

$\leftrightarrow$ any non-empty chain of subsets of $X$ has a maximal member $\leftrightarrow$ any non-empty chain of subsets of $X$ has a minimal member.

Proof. The equitalence of the last two is easy.

Suppose $|X| \in \Delta_{2}$, and let $C$ be any non-empty chain of subsets of $X$. For $\xi \in X$ let $A_{\xi}=\{c \in C: \xi \in c\}$.

Define the partition $\pi$ on $X$ by

$$
\xi, \eta \text { are in the same member of } \pi \leftrightarrow A_{\xi}=A_{\eta} \text {. }
$$

Let $(\xi)$ be the member of $\pi$ containing $\xi . \pi$ is ordered by

$$
(\xi) \leqslant(\eta) \quad \text { if } \quad A_{\xi} \subset A_{\eta}
$$

Since $C$ is a chain, $(\pi,<)$ is an ordered partition of $X$. By definition of $\triangle_{2}, \pi$ is finite. If $C=\{\emptyset\}, \emptyset$ is maximal in $C$. Otherwise, as $C \neq \varnothing$, there is a $(\xi) \epsilon \pi$ such that $A_{\xi} \neq \varnothing$. Let $(\xi)$ be the least such, and let $c \in A_{\xi}$. It is clear that $c$ is a maximal member of $C$.

Conversely, suppose that any non-empty chain of subsets of $X$ has a maximal element. Let $(\pi,<)$ be an ordered partition of $X$. To show that it is finite we need only show that $<$ and $>$ are well-orderings. Let $P$ be a non-empty subset of $\pi$, and let $C=\{\bigcup I: I$ a proper initial segment of $(P,<)\}$. Then $C$ is a chain of subsets of $X$, and is non-empty, as $\emptyset \in C$. By our hypothesis, $C$ has a maximal member. This gives a maximal proper initial segment of $(P,<)$, and hence a maximal member of $P$.

Similarly, by considering final segments, we see that $P$ has a minimal member.

LEMTMA 2. $x \in \Delta_{4} \leftrightarrow 2^{x} \in \Delta$.

This is due to Kuratowski, and is proved on pages 94, 95 of [18]. Thus $\Delta_{4}$ comprises just those cardinals finite in sense III of Levy. We should like to have the analogue of this for $\Delta_{2}$ and $\Delta_{3}$, but suspect that it is in fact false (though it holds in all the models considered here).

Lemara 3. $\omega \subset \Delta_{1} \subset \Delta_{2} \subset \Delta_{4} \subset \Delta_{5} \subset \Delta, \Delta_{2} \subset \Delta_{3} \subset \Delta$.

Proof. Le'vy showed in [8] Theorem 1 that $\omega \subset \Delta_{1} \subset \Delta_{2} \subset \Delta_{4} \subset \Delta$, so it remains to show that $\Delta_{4} \subset \Delta_{5} \subset \Delta$ and $\Delta_{2} \subset \Delta_{3} \subset \Delta$. The only point presenting any difficulty is $\Delta_{4} \subset \Delta_{5}$.

Suppose that $x+1 \leqslant^{*} x,|X|=x$, and let $f \operatorname{map} X$ onto $X \cup\{X\}$. Let $X_{n}=f^{-(n+1)}(\{X\})$. Then every $X_{n}$ is non-empty, as $f$ is "onto", and all the $X_{n}$ 's are disjoint and contained in $X$. Hence $\kappa_{0} \leqslant *|X|$. 
Lemma 4 (Tarski [9], page 304,24 ). If $x+y \leqslant^{*} x+z$ there are $a, b$ such that $y=a+b, b \leqslant^{*} z$, and $x+a \leqslant^{*} x$.

LEMMA 5. $x \in \Delta_{5} \leftrightarrow$ (for all $p, q, p \leqslant * q \leftrightarrow x+p \leqslant^{*} x+q$ ).

Proof. Suppose $x \in \Delta_{5}$, and $x+p \leqslant^{*} x+q$. By Lemma 4 there are $a, b$ such that $p=a+b, b \leqslant^{*} q, x+a \leqslant * x$. Since $x+1 \star^{*} x, a=0$. Therefore $p=b \leqslant * q$, as desired.

Conversely, if $x \notin \Delta_{5}, x+1 \leqslant * x$, so there are $p$, namely $p=1$, $q=0$, such that $p \leqslant * q$ and $x+p \leqslant * x+q$.

LEMMA 6. Let $q(X)=$ the set of all finite sequences of members of $X$ with distinct entries. $q(|X|)=|q(X)|$. If $x \in \Delta, \Delta_{3}$ respectively, then $q(x) \in \Delta, \Delta_{3}$, and if $x$ is infinite, $q(x) \notin \Delta_{5}$.

Proof. This lemma for $\Delta$ is due to Tarski. See [7], page 225, lines 16-20. Suppose that $(A,<)$ is a well-ordered (ordered respectively) subset of $q(\bar{X})$, where $|X|=x$.

Let $A_{n}=\{\xi \in A: \xi$ has length $n\}$. Assume the result of Theorem 1 (vi) below (in whose proof we do not use this lemma) that $\Delta$ and $\Delta_{3}$ are closed under $x$. Then $\left|X^{n}\right| \in \Delta,\left(\left|X^{n}\right| \in \Delta_{3}\right.$ respectively). Therefore each $A_{n}$ is finite, and $A$ can be well-ordered. The set of entries of members of $A$ can be well-ordered by first oceurrence, so as $|X| \epsilon \Delta$, is finite.

Therefore $A$ too is finite, as desired.

If $X$ is infinite, map $q(X)$ onto $q(X) \cup\left\{\eta_{0}\right\}\left(\eta_{0} \notin q(X)\right)$ thus

$$
\left(\xi_{0}, \xi_{1}, \ldots, \xi_{n-1}\right) \rightarrow\left(\xi_{0}, \xi_{1}, \ldots, \xi_{n-2}\right) \text { if } n>0 \text {, }
$$

( ) $\rightarrow \eta_{0}(()$ is the empty sequence).

Since $X$ is infinite, for any $\left(\xi_{0}, \xi_{1}, \ldots, \xi_{n-1}\right)$ there is a $\xi_{n} \neq \xi_{i}$, any $i$, and so the map is "onto".

LEMMA 7. If $\Delta_{4} \subset \Delta_{3}$ then $\Delta_{2}=\Delta_{4}$.

Proof. Let $x \in \Delta_{4}, x=|X|$, and suppose $(\pi,<)$ is an ordered partition of $X$. Then $|\pi| \leqslant *|X|$. Since

$$
\kappa_{0} \mathbb{K}^{*} x, \quad \kappa_{0} \mathbb{K}^{*}|\pi| \text {. }
$$

Hence $|\pi| \epsilon \Delta_{4} \subset \Delta_{3}$, by hypothesis. Now $(\pi,<)$ is an ordered subset of $\pi$. Therefore $\pi$ is finite, and $x \in \Delta_{2}$.

CoRollary. If $\Delta_{3}=\Delta$, then $\Delta_{2}=\Delta_{4}$.

The converse of this corollary does not hold, for in Halpern-Levy's model ([1], page 136), the ordering principle holds, so $\Delta_{2}=\Delta_{3}=\omega$, any infinite set has a countable partition, giving $\Delta_{4}=\omega$, but there is an infinite set with no countable subset, so $\omega \neq \Delta$.

§ 3. Closure under various operations. We now investigate the question of the closure of the classes under unions, disjoint unions,,$+ \times$, and $\leqslant$. The positive results are set out in the following theorem.
THEOREM 1. (i) All of $\omega, \Delta, \Delta_{1}, \ldots, \Delta_{5}$ are closed under $\leqslant$.

(ii) $\omega, \Delta_{2}, \Delta_{4}$ are closed under unions.

(iii) $\Delta_{3}, \Delta$ are closed under disjoint unions.

(iv) $\Delta_{5}$ is closed under + .

(v) $\Delta_{1}$ is closed under + if and only if $\omega=\Delta_{1}$.

(vi) $\omega, \Delta_{2}, \Delta_{3}, \Delta_{4}, \Delta$ are closed under,$+ \times$.

Proof. (i) is obvious in all our cases, but is none the less important. For example, the class $\{x: x<2 x\}$ corresponding to definit on $V$ of Lery is not necessarily closed under $\leqslant$.

(ii) This is well known for $\omega$. Let $|X| \in \Delta_{2}\left(\Delta_{4}\right)$, and let $\xi \in X \rightarrow|\xi|$ $\epsilon \Delta_{2}\left(\Delta_{4}\right)$. Suppose that $(\pi,<)$ is an ordered (well-ordered respectively) partition of $\cup X$. For each $\xi \in X, \xi \subset \cup X$, and we get the induced partition of $\xi, \pi_{\xi}=\{\xi \cap A: A \epsilon \pi$ and $\xi \cap A \neq \emptyset\}$, also ordered (well-ordered).

Since $|\xi| \epsilon \Delta_{2}\left(\Delta_{4}\right)$, each $\pi_{\xi}$ is finite. The finite subsets of an ordered (well-ordered) set can be ordered (well-ordered). Let $<_{1}$ order (well-order) the finite subsets of $\pi$. Define the partition $\varrho$ of $X$ by

$\xi, \eta$ are in the same member of $\varrho$ if for $A \in \pi$,

$$
\xi \cap A=\emptyset \leftrightarrow \eta \cap A=\varnothing .
$$

$\varrho$ has the ordering (well-ordering) induced by $<_{1}$.

Since $|X| \in \Delta_{2}\left(\Delta_{4}\right), \varrho$ is finite. Hence only finitely many members of $\pi$ appear, and this means that there are only finitely many of them.

(iii) Let $X$ be disjointed, $|X| \epsilon \Delta_{3}(\Delta)$ and $\xi \epsilon X \rightarrow|\xi| \epsilon \Delta_{3}(\Delta)$. Let $(A,<)$ be an ordered subset of $\bigcup X$ (well-ordered respectively).

For each $\xi \in X$, let $A_{\xi}=A \cap \xi$. Since $|\xi| \epsilon \Delta_{3}(\Delta), A_{\xi}$ is finite.

Let $B=\left\{\xi \in X: A_{\xi} \neq \varnothing\right\}$. Then $B$ is ordered by $\xi<\eta$ if the first member of $A_{\xi}$ precedes the first member of $A_{\eta}$ in $(A,<)$. This defines a linear (well-) ordering of $B$ because $X$ is disjointed. Hence $B$ is finite, as $|X| \in \Delta_{3}(\Delta)$.

Therefore $A$ is also finite, as desired.

(iv) Let $x, y \in \Delta_{5}$. Suppose that $(x+y)+p \leqslant *(x+y)+q$.

By Lemma $5, y+p \leqslant^{*} y+q$ and $p \leqslant^{*} q$. Hence, again by Lemma 5 , $x+y \in \Delta_{5}$.

(v) If $x \in \Delta_{1}-\omega$, then $x+x \notin \Delta_{1}$.

(vi) Follows from (i), (ii), and (iii), since $x+y \leqslant x y$.

Next we have the following negative result, which answers the remaining closure questions about the classes.

THeorem 2. (i) If $\omega \neq \Delta, \Delta$ is not closed under unions.

(ii) If $\omega \neq \Delta_{3}, \Delta_{3}$ is not closed under unions.

(iii) If $\mathrm{ZF}$ is consistent, so is $\mathrm{ZF}+\Delta_{5}$ is not closed under $\times$.

Proof. (i) and (ii). By Lemma 6 there is an $X$ such that $|X| \in \Delta-\Delta_{5}$ $\left(\Delta_{3}-\Delta_{5}\right.$ respectively), and so $|X| \in \Delta-\Delta_{4}\left(\Delta_{3}-\Delta_{4}\right.$ respectively). 2 - Fundamenta Mathematicae LXXXrV 
Let $X=\bigcup\left\{X_{i}: i \in \omega\right\}, X_{i}$ disjoint and non-empty. If $\xi \in X_{i}$, let $Y_{\xi}=X \cup\{i\}$.

For each $\xi \in X,\left|Y_{\xi}\right| \in \Delta\left(\Delta_{3}\right)$, and $|X| \in \Delta\left(\Delta_{3}\right)$. But $\omega \subset \bigcup\left\{Y_{\xi}: \xi \in X\right\}$.

Hence $\Delta, \Delta_{3}$ are not closed under unions.

(iii) TVe first prove the following lemma, which will be used in a number of cases where we wish to show that a particular cardinal is in $\Delta_{5}$.

LeMcua 8. If $X=\bigcup\left\{X_{i}: i \in \omega\right\}$ where each $\left|X_{i}\right| \in \Delta_{4}$, and $|X| \notin \Delta_{5}$, then there is a map from $X$ onto $X \cup\{\alpha\}(\alpha \notin X)$, and an increasing function $n$ from $\omega$ to $\omega$ such that for each $i$,

$$
f^{-(i+1)}(\{\alpha\}) \subset X_{n(i)} \text {. }
$$

Proof. As $|X| \notin \Delta_{5}$ there is a map $g$ from $X$ onto $X \cup\{\alpha\}$. Define $A_{i}$, $n(i), m(i)$ in the following way, so that $A_{i} \neq \varnothing$, each $i, n$ is increasing, $m(i)>0$, each $i$.

$n(0)$ is the least $i$ such that $g^{-1}(\{\alpha\}) \cap X_{i} \neq \emptyset$

$m(0)=1$,

$A_{0}=g^{-1}(\{\alpha\}) \cap X_{n(0)}$

Otherwise suppose that $A_{i}, n(i), m(i)$ are defined for $i<k$. Then

$$
\bigcup\left\{g^{-j}\left(A_{k-1}\right): j>0\right\} \mid \notin \Delta_{5} \quad \text { (use } g \text { to show this), }
$$

and

$$
\text { UU }\left\{X_{j}: j \leqslant n(k-1)\right\} \mid \in \Delta_{5} \quad \text { (by Theorem } 1 \text { (iv)). }
$$

Hence

$$
\bigcup\left\{g^{-j}\left(A_{k-1}\right): j>0\right\} \not \subset \bigcup\left\{X_{j}: j \leqslant n(k-1)\right\} .
$$

So for some $n>n(k-1), m>0$,

$$
g^{-m}\left(A_{k-1}\right) \cap X_{n} \neq \varnothing \text {. }
$$

Let $n(k)$ be the least possible such $n$, and for that value of $n, m(k)$ the least possible $m$. Let $A_{k}=g^{-m(k)}\left(A_{k-1}\right) \cap X_{n(k)}$.

Thus $A_{k}, n(k), m(k)$ are all defined, each $k$, and $g^{m(k)}$ maps $A_{k}$ into $A_{k-1}$ (not necessarily "onto").

Let $B_{k}=\left\{\xi \in A_{k}\right.$ : there are arbitrarily large $n$ such that for some $\left.\eta \in \bigcup\left\{A_{j}: j \in \omega\right\}, g^{n}(\eta)=\xi\right\}$.

Define $f$ on $X$ thus. If $\xi \in B_{k}, f(\xi)=g^{m(k)}(\xi) ; f(\xi)=\xi$ otherwise. Then all the required conditions are satisfied, except possibly that $f$ is "onto".

If $\xi \notin B_{k}$, each $k$, then $\xi=f(\xi)$, so $\xi \in$ image $f$.

Suppose that $\xi \in B_{k}$, or that $\xi=\alpha$, and $\xi \notin$ image $f$. Then for each $\eta \in A_{k+1}$ (or $A_{0}$ if $\xi=\alpha$ ) such that $f(\eta)=\xi, \eta \notin B_{k+1}$.

That is, for every such $\eta \in A_{k+1}$ there is a least $k(\mu) \epsilon \omega$ such that $\zeta \in \bigcup\left\{A_{i}: i \in \omega\right\} \rightarrow g^{k(\mu)}(\zeta) \neq \xi$.
Arbitrarily large values of $k(\mu)$ must appear, by definition of $B_{k}$. Hence $s_{0} \leqslant{ }^{*}\left|A_{k+1}\right|$. This is contrary to $\left|X_{n(k+1)}\right| \in \Delta_{4}$.

We now give a Fraenkel-Mostowski model in which $\Delta_{5}$ is not closed under $x$. The ZF consistency result then follows by the Jech-Sochor Theorem [5], since the statement in question is

但 $(X, Y, f) \quad(f$ maps $X \times Y$ onto $X \times Y \cup\{X \times Y\}$ and for all $g$ $C(X \cup Y)^{2}$, if $g$ is a mapping from $X \cup Y$ onto $X \cup Y$, then $g$ is $\left.1-1\right)$.

We suppose then that we have a model $\mathfrak{M}$ of $\mathrm{FM}$ (= ZF modified so as to allow the existence of "urelemente"; one only needs to alter the axiom of extensionality) in which $U$, the class of all urelemente, is a set of cardinal $\aleph_{a}$. We suppose that the axiom of choice holds in $\mathfrak{M}$.

Since $U$ is countable, it may be indexed by $\Pi$, the set of all finite sequences of 0 's and 1 's, so we let $U=\left\{u_{\pi}: \pi \in \Pi\right\}$. For convenience we write $\left[\alpha_{0} \alpha_{1} \ldots \alpha_{i}\right]$ instead of $u_{\left\langle\alpha_{0}, \alpha_{1}, \ldots, \alpha_{i}\right\rangle}$.

Let

$$
V=\left\{u_{\pi}: \pi \text { begins with a } 0\right\} \text { and } W=\left\{u_{\pi}: \pi \text { begins with a } 1\right\} \text {. }
$$

$G$ is the group of all permutations of $U$ which preserve $V, W$ and the lengths of the sequences.

Define $f: V \times W \rightarrow V \times W \cup\{\varnothing\}$ thus

$$
\begin{gathered}
f\left(\left[0 \alpha_{1}\right],\left[1 \beta_{1}\right]\right)=\emptyset, \\
f\left(\left[0 \alpha_{1} \alpha_{2} \ldots \alpha_{i+2}\right],\left[1 \beta_{1} \beta_{2} \ldots \beta_{i+2}\right]\right)=\left(\left[0 \alpha_{1} \alpha_{2} \ldots \alpha_{i} \beta_{i+1}\right],\left[1 \beta_{1} \beta_{2} \ldots \beta_{i} \alpha_{i+1}\right]\right)
\end{gathered}
$$

and

$$
f\left(\pi_{1}, \pi_{2}\right)=\left(\pi_{1}, \pi_{2}\right) \quad \text { otherwise . }
$$

Thus for members of $V \times W$ where each half is of equal length $>2$, $f$ deletes the last elements, and interchanges the last but one.

Clearly $f$ maps $\nabla \times W$ onto $V \dot{\times} W \cup\{\varnothing\}$.

If $\sigma \in G$ and $\xi \in \mathfrak{M}, \sigma \xi$ is defined by transfinite induction on rank $\xi$ thus : $-\sigma \xi=\{\sigma \eta: \eta \in \xi\}$.

For each $\xi$ we then let

$$
H(\xi)=\{\sigma \in G: \sigma \xi=\xi\} \text { and } \quad K(\xi)=\{\sigma \in G: \eta \ddot{\epsilon} \xi \rightarrow \sigma \eta=\eta\} .
$$

$\widetilde{F}$ is the filter of subgroups of $G$ generated by $\{H(u): u \in U\}$ and $H(f)$.

$\mathfrak{N}$ is the resulting Fraenkel-Mostowski model. That is to say

$$
\xi \in \mathfrak{R} \quad \text { if and only if } \xi \subset \mathfrak{N} \text { and } H(\xi) \in \mathfrak{F} .
$$

That $\mathfrak{N}$ is a model of $F M$, with membership relation just the restriction of that on $\mathfrak{M}$ to $\mathfrak{N}$ is proved, for example, by Mostowski in [11].

Clearly $U, V, W, f \in \mathfrak{R}$. Let $v=|V|^{\Re}, w=|W|^{\Re}$. Since $f \in \mathfrak{N}, v w \notin \Delta_{t}$ 
in $\mathfrak{N}$. We show that $v$ (and similarly $w$ ) $\in \Delta_{5}$ in $\mathfrak{R}$. It follows that $\Delta_{5}$ is not closed under $x$ in $\mathfrak{\Re}$.

Since each member of $G$ preserves the lengths of members of $\Pi$, the sequence $\left(U_{0}, U_{1}, \ldots\right)$ is in $\mathfrak{N}$, where $U_{i}$ is the set of members of $U$ of length $i$. Of course each $U_{i}$ is finite.

Suppose that $v \notin \Delta_{5}$. By Lemma 8 there is a map $g$ in $\mathfrak{N}$ from $V$ onto $\nabla \cup\{\varnothing\}$, and an increasing map $n: \omega \rightarrow \omega$ such that for each $i$, every member of $f^{-(i+1)}(\{\emptyset\})$ has length $n(i)$.

Since $g \in \mathfrak{R}$ there is a finite $A \subset U$ such that

$$
H(g) \supset H(f) \frown K(A) \text {. }
$$

Let $i$ be the least integer $>0$ such that every member of $A$ has length $\leqslant i$. Let $g(\alpha)=\beta$, where length $\alpha=k$, length $\beta=j$, and $k>j>i$.

Then if $\sigma \in H(g) \cap H(\alpha), \beta=g(\alpha)=g(\sigma \alpha)=\sigma g(\alpha)=\sigma \beta$. This shows that $H(g) \frown H(\alpha) \subset H(\beta)$, so

$$
K(A) \cap H(f) \cap H(\alpha) \subset H(\beta) .
$$

To obtain the desired contradiction, we find a $\sigma \in H(f)$ which fixes all members of $V$ of length $\neq j$, all members of $W$ of length $\leqslant i$, and moves every member of $V$ of length $j$.

Such a $\sigma$ will be in $K(\dot{A}) \cap H(f) \cap H(\alpha)$ but not in $H(\beta)$. Let

$$
\begin{aligned}
\sigma\left[0 \gamma_{1} \gamma_{2} \ldots \gamma_{j-1}\right] & =\left[0 \gamma_{1} \gamma_{2} \ldots \gamma_{j-2} 1-\gamma_{j-1}\right], \\
\sigma\left[1 \delta_{1} \delta_{2} \ldots \delta_{l}\right] & =\left[1 \delta_{1} \delta_{2} \ldots \delta_{j-2} 1-\delta_{j-1} \delta_{j} \ldots \delta_{l}\right], \text { if } l>j-1,
\end{aligned}
$$

and $\sigma$ fix everything else.

Then $\sigma$ fixes lengths of sequences, and $\nabla, W$, so lies in $G$. $\sigma$ also fixes every member of $V$ of length $\neq j$ and every member of $W$ of length $\leqslant i$ (as $i<j$ ), and moves every member of $\nabla$ of length $j$.

It remains to show that $\sigma \in H(f)$. This is the same as saying that $f(\sigma \pi)=\sigma f(\pi)$, each $\pi \in \nabla \times W$.

Let $\pi=\left(\pi_{1}, \pi_{2}\right)$. If $\pi_{1}$ and $\pi_{2}$ have different lengths, $f(\pi)=\pi$, and $\sigma \pi_{1}, \sigma \pi_{2}$ also have different lenths, so $f(\sigma \pi)=\sigma \pi=\sigma f(\pi)$.

Suppose therefore that they have equal lengths, $l+1$. Let

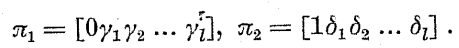

(I) $l+1<j$. Then $\sigma$ fixes $\pi$ and $f(\pi)$, so $\sigma f(\pi)=f(\pi)=f(\sigma \pi)$.

(II) $l+1=j . \quad f(\tau)=\left(\left[0 \gamma_{1} \gamma_{2} \ldots \gamma_{j-3} \delta_{j-2}\right],\left[1 \delta_{1} \delta_{2} \ldots \delta_{j-3} \gamma_{j-2}\right]\right)$ and as $j-1<j, \sigma f(\pi)=f(\pi)$.

$$
\sigma \pi=\left(\left[0 \gamma_{1} \gamma_{2} \ldots \gamma_{j-2} 1-\gamma_{j-1}\right],\left[1 \delta_{1} \delta_{2} \ldots \delta_{j-1}\right]\right),
$$

and so

$$
f(\sigma \pi)=\left(\left[0 \gamma_{1} \gamma_{2} \ldots \gamma_{j-3} \delta_{j-2}\right],\left[1 \delta_{1} \gamma_{2} \ldots \delta_{j-3} \gamma_{j-2}\right]\right)=\sigma f(\pi) .
$$

(III) $l+1=j+1$.

and

$$
f(\pi)=\left(\left[0 \gamma_{1} \gamma_{2} \ldots \gamma_{j-2} \delta_{j-1}\right],\left[1 \delta_{1} \delta_{2} \ldots \delta_{j-2} \gamma_{j-1}\right]\right),
$$

Thus

$$
\sigma f(\pi)=\left(\left[0 \gamma_{1} \gamma_{2} \ldots \gamma_{j-2} 1-\delta_{j-1}\right],\left[1 \delta_{1} \delta_{2} \ldots \delta_{j-2} \gamma_{j-1}\right]\right) .
$$

(IV) $l+1>j+1$.

$$
\begin{aligned}
f(\sigma \pi) & =f\left(\left[0 \gamma_{1} \gamma_{2} \ldots \gamma_{j}\right],\left[1 \delta_{1} \delta_{2} \ldots 1-\delta_{j-1} \delta_{j}\right]\right) \\
& =\left(\left[0 \gamma_{1} \gamma_{2} \ldots \gamma_{j-2} 1-\delta_{j-1}\right],\left[1 \delta_{1} \gamma_{2} \ldots \delta_{j-2} \gamma_{j-1}\right]\right)=\sigma f(\pi) .
\end{aligned}
$$

and

$$
f(\pi)=\left(\left[0 \gamma_{1} \gamma_{2} \ldots \gamma_{l-2} \delta_{l-1}\right],\left[1 \delta_{1} \delta_{2} \ldots \delta_{l-2} \gamma_{l-1}\right]\right)
$$

Thus

$$
\sigma f(\pi)=\left(\left[0 \gamma_{1} \gamma_{2} \ldots \gamma_{l-2} \delta_{l-1}\right],\left[1 \delta_{1} \delta_{2} \ldots 1-\delta_{j-1} \ldots \delta_{l-2} \gamma_{l-1}\right]\right) .
$$

$f(\sigma \pi)=f\left(\left[0 \gamma_{1} \gamma_{2} \ldots \gamma_{l}\right],\left[1 \delta_{1} \delta_{2} \ldots 1-\delta_{j-1} \ldots \delta_{l}\right]\right)$

$$
=\left(\left[0 \gamma_{1} \gamma_{2} \ldots \gamma_{l-2} \delta_{l-1}\right],\left[1 \delta_{1} \delta_{2} \ldots 1-\delta_{j-1} \ldots \delta_{l-2} \gamma_{l-1}\right]\right)=\sigma f(\pi) .
$$

To obtain the ZF consistency result as stated we use the Jech-Sochor Theorem [5], as mentioned abote. Pincus [14] 2B7 could also be used.

§ 4. Now suppose that $|X| \in \Delta_{1}-\omega$. We define the 2 - valued function $\mu_{n}$ from $P\left(X^{n}\right)$ to $\{0,1\}$, by induction on $n$.

$$
\begin{array}{lrl}
n=0 & \mu_{0}(\varnothing)=0, & \\
& \mu_{0}(\{()\})=1 & (() \text { is the empty sequence) } . \\
n+1 & \mu_{n+1}(A)=0 & \text { if }\left\{\xi \in X: \mu_{n}\left(A_{\xi}\right)=1\right\} \text { is finite } \\
& \mu_{n+1}(A)=1 & \text { otherwise, }
\end{array}
$$

where $A_{\xi}=\left\{\eta \in X^{n}:\langle\xi\rangle \cap \eta \in A\right\}$.

Let $X_{n}=\left\{\left(\xi_{0}, \xi_{1}, \ldots, \xi_{n-1}\right) \in X^{n}: \xi_{0}=\xi_{1}\right\}$.

The proof of the following lemma is very simple, and is omitted.

LEMMA $9 . \mu_{n}$ is a finitely additive measure on $X^{n}$, vanishing on singletons when $n>0$, and satisfying $\mu_{n}\left(X^{n}\right)=1, \mu_{n}\left(X_{n}\right)=0$.

Let $\sigma$ be a permutation of $\{0,1, \ldots, n-1\}$. If $A \subset X^{n}$ we let $A_{\sigma}=\left\{\xi_{\sigma}: \xi \in A\right\} \quad$ where $\left(\xi_{0}, \xi_{1}, \ldots, \xi_{n-1}\right)_{\sigma}=\left(\xi_{\sigma 0}, \xi_{\sigma 1}, \ldots, \xi_{\sigma(n-1)}\right)$.

LEMMa 10. For any $A \subset X^{n}$ and permutation $\sigma, \mu_{n}(A)=\mu_{n}\left(A_{\sigma}\right)$.

Sketch of Proof. Firstly we may suppose that $\sigma$ is a transposition of adjacent elements, as such transpositions generate the symmetric group. One performs the proof in three stages, corresponding respectively to the possibilities $n=2, \sigma=(01) ; n \geqslant 2, \sigma=(01) ; \sigma=(i \quad i+1)$. 
THEOREM 3. If $X$ is infinite and $f$ is a function on $[X]^{n}$ such that $\emptyset \neq f(A) \subset A, f(A) \neq A$, for each $A \in[X]^{n}$, then $|X| \notin \Delta_{1}$.

Proof. Suppose $|X| \epsilon \Delta_{1}$. Then $\mu_{n}$ can be defined. Let

$C=\left\{\left(\xi_{0}, \xi_{1}, \ldots, \xi_{n-1}\right): \xi_{j}\right.$ all distinct, and for some $i<n$,

$$
\left.\left\{\xi_{0}, \xi_{1}, \ldots, \xi_{i-1}\right\}=f\left(\left\{\xi_{0}, \xi_{1}, \ldots, \xi_{n-1}\right\}\right)\right\} \text {. }
$$

Then $\mu_{n}\left(C_{0 n-1}\right)=\mu_{n}(C)$ by Lemma 10. It is clear that $C \cap C_{0 n-1}=\varnothing$ as $i \neq 0$. Hence $\mu_{n}\left(C_{0 n-1}\right)=\mu_{n}(C)=0$.

Let $X_{n}^{\prime}=\left\{\left(\xi_{0}, \xi_{1}, \ldots, \xi_{n-1}\right)\right.$ : for some $\left.i \neq j, \xi_{i}=\xi_{j}\right\}$. By Lemmas 9 and 10

$$
\mu_{n}\left(X_{n}^{\prime}\right)=\mu_{n}\left(\bigcup\left\{\left(X_{n}\right)_{\sigma}: \sigma \text { a permutation of } n\right\}\right)=0 .
$$

Therefore

$$
\begin{aligned}
\mu_{n}\left(X^{n}\right) & =\mu_{n}\left(\bigcup\left\{C_{\sigma}: \sigma \text { a permutation of } n\right\}\right)+\mu_{n}\left(X_{n}^{\prime}\right) \\
& =0+0=0, \quad \text { a contradiction. }
\end{aligned}
$$

COROLLARY. If for some $n>1,[X]^{n}$ has a choice function, then $X$ is finite or $|X| \notin \Delta_{1}$.

LEMMA. 11. If any two members of $\Delta$ are comparable, and $|e(X)| \epsilon \Delta$, then $e(X)-\{\emptyset\}$ has a choice function, where $e(X)=\{A \subset X: A$ finite $\}$.

Proof. Let $e^{*}(X)=\{(A, n): A \in e(X), n<|A| !\} . e^{*}(X)$ is designed to be the same "size" as $q(X)$ (the set of finite $1-1$ sequences of members of $X$; see Lemma 6 ) at each stage. For this reason we put in $|A|$ ! copies of each $A$ corresponding to the $|A|$ ! different orderings of $A$. It is easily seen that $|e(X)| \epsilon \Delta \rightarrow\left|e^{*}(X)\right| \epsilon \Delta$.

By the comparability of any two members of $\Delta$,

$$
\left|e^{*}(X)\right| \leqslant|q(X)| \quad \text { or } \quad|q(X)| \leqslant\left|e^{*}(X)\right| .
$$

Let $f$ map $e^{*}(X) 1-1$ into $q(X)$ (or vice versa), and let $A \in e(X)$. We show how to choose effectively in terms of $f$ a $B \supset A$ such that $f$ maps $e^{*}(B)$ 1-1 onto $q(B)$ (or vice versa), and $B$ is finite.

Let $A_{0}=A \cdot A_{n+1}$ is the union of $A_{n}$ and $\{\xi: \xi$ is the set of entries of a member of $\left.f^{\prime \prime}\left(e^{*}\left(A_{n}\right)\right)\right\}$, or $\left\{\xi\right.$ : for some $\left.i,(\xi, i) \epsilon f^{\prime \prime}\left(q\left(A_{n}\right)\right)\right\}$ if $f$ goes the other way round.

As $|e(X)| \in \Delta, A_{n}=A_{n+1}$ for some $n$, and this is our $B . f$ is then 1-1 from $e^{*}(B)$ into $q(B)$ (or vice versa), and as $\left|e^{*}(B)\right|=|q(B)|$ it is also "onto".

Thus by taking $f^{-1}$ in place of $f$ in the second case, we have an effectively determined finite $B \supset A$, and a 1-1 map $f$ from $e^{*}(B)$ onto $q(B)$. We show that when $A \neq \emptyset$ this gives an effectively determined $g(A) \in A$, which is what is required.
Let $C$ be the closure of $\{(A, 0)\}$ under the operations

(i) if $(\xi, i) \in C$, so is $(\xi, j)$, any $j<|\xi|$ ! and

(ii) if $(\xi, i) \in C, P(\eta) \times\{0\} \subset C$ where $\eta$ is the set of entries of $f((\xi, i))$.

It is clear that $C$ can be effectively ordered, and that $O \subset e^{*}(B)$. Also $f$ maps $C$ 1-1 onto $\bigcup\{q(\eta):(\eta, 0) \in C\} . g(A)$ is the first entry of the first member of $q(A)$ in the ordering of the image of $C$. Thus $g$ is a choice function for $e(\bar{X})-\{\varnothing\}$, as desired.

COROLLARY. If any two members of $\Delta$ are comparable, then $\omega=\Delta_{1}$.

Proof. Use Theorem 3 and Lemma 11.

We mention another way in which this corollary can be derived, without giving details. First one proves the following.

If any two members of $\Delta$ are comparable, and $|X| \epsilon \Delta_{4}, X$ infinite, there is a permutation of $X$ moving infinitely many points.

To derive the corollary from this, suppose $X$ is infinite, $|X| \epsilon \Delta_{1}$. Then $|X| \in \Delta_{4}$, so there is a permutation $\sigma$ of $X$ moving infinitely many points. Since $|X| \in \Delta$, each cycle of $X$ under $\sigma$ is finite. There are thus infinitely many finite cycles with at least two members. Let $Y$ be a subset of $X$ which chooses a member from each such cycle (using Lemma 11). Then $Y$ and $X-Y$ are infinite disjoint subsets of $X$, contrary to $|X| \epsilon \Delta_{1}$.

It seems likely that an argument along the lines of Theorem 3 , using Lemma 8, will show that if $x \in \Delta_{1}, e(x) \in \Delta_{5} .(e(X)$ is the set of finite subsets of $X$.) At present the best we have is the following.

THEOREM 4. If $x \in \Delta_{2}-\omega, e(x) \in \Delta_{3}-\Delta_{4}$.

Proof. Let $x \in|X|$. Of course $e(x) \notin \Delta_{4}$, as $e(X)=\bigcup\left\{[X]^{n}: n \epsilon \omega\right\}$. Suppose $(A,<)$ is an infinite ordered subset of $e(X)$. Then $\cup A$ is infinite.

For $\xi \in \cup A$, let $n(\xi)$ be the least $n$ such that for some $B \in A, \xi \in B$ and $|B|=n$. As $x \in \Delta_{4}, n(\xi)$ is bounded for $\xi \in \cup A$. Thus for some $n$, and some infinite $B \subset A, B \subset[X]^{n}$. Hence $X^{n}$ has an infinite ordered partition, as it can be mapped onto $[X]^{n}$. This is contrary to Theorem 1 (vi) that $\Delta_{2}$ is closed under $\times$

\$ 5. Five models. The models we use in this section are already known; but we shall be concerned with properties of them not previously studied. For example it was not known before that in three of them $|\Delta|=2^{\boldsymbol{N}_{0}} . \mathfrak{N}_{1}$ is due to Halpern and Levy ([1], page 136), $\mathfrak{N}_{2}$ and $\mathfrak{N}_{3}$ are due to Mostowski ([10] and [11] respectively), and $\mathfrak{R}_{4}$ is due to Gauntt [3]. $\mathfrak{N}_{5}$ is only a slight modification of Levy's model $\mathfrak{M}^{\prime},[8]$ page 11 . When the model we are using contains urelemente, we appeal to Pincus [14] 2B7 to give the corresponding $\mathrm{ZF}$ result.

"Combinations" of the models give all but ten of the possible combinations of equality and strict inclusion in Lemma 3 (subject to the restrictions of $\S 2$ and $\S 3$ ). For example, $\omega=\Delta_{1}=\ldots=\Delta$ occurs wheneter 
we have DC. To obtain a model for $\omega=\Delta_{1} \neq \Delta_{2} \neq \Delta_{4} \neq \Delta_{5} \neq \Delta, \Delta_{2} \neq \Delta_{3}$ $\neq \Delta$, combine the methods of (i), (iii), and (iv).

THEOREM 5. If ZFF is consistent, then so are each of the following

(i) $\mathrm{ZF}+\omega=\Delta_{1}=\Delta_{2}=\Delta_{4} \neq \Delta_{5} \neq \Delta, \Delta_{2}=\Delta_{3} \neq \Delta$,

(ii) $\mathrm{ZF}+\omega=\Delta_{1}=\Delta_{2}=\Delta_{4} \neq \Delta_{5} \neq \Delta, \Delta_{2} \neq \Delta_{3}=\Delta$,

(iii) $\mathrm{ZF}+\omega=\Delta_{1}=\Delta_{2} \neq \Delta_{4}=\Delta_{5} \neq \Delta, \Delta_{2}=\Delta_{3} \neq \Delta$,

(iv) $Z F+\omega=\Delta_{1} \neq \Delta_{2}=\Delta_{4} \neq \Delta_{5} \neq \Delta, \Delta_{2} \neq \Delta_{3}=\Delta$,

(v) $\mathrm{ZF}+\omega \neq \Delta_{1} \neq \Delta_{2}=\Delta_{4} \neq \Delta_{5} \neq \Delta, \Delta_{2} \neq \Delta_{3}=\Delta$.

Pro of. $\mathfrak{N}_{1}$ is Halpern and Lery's model, defined on page 136 of [1]. It is obtained from the ground model $\mathfrak{M}$ (a countable transitive model of $\mathrm{ZFC}+\mathrm{GCH}$ ) by adjoining a set $b$ of generic reals, $a_{i}, i \in \omega$, but not their indexing by $\omega$.

$\mathfrak{R}_{2}$ is the following Fraenkel-Mostowski model. We use the same notation for FM models as in $\S 3 . U=\left\{u_{i j}: i \in \omega, j \in 2\right\}, U_{i}=\left\{u_{i 0}, u_{i 1}\right\}$. $G$ is the group of all permutations of $U$ which fix each $U_{i}$. $₹$ is the filter of subgroups of $G$ generated by $\{H(u): u \in U\}$. This model was studied in [19].

$\mathfrak{N}_{3}$ is Mostowski's model of [11], used by Levy in [8]. $U=\left\{u_{q}: q \in Q\right\}$, where $Q$ is the set of rational numbers. $G$ is the group of all orderpreserving permutations of $U$, and $\mathfrak{F}$ is generated by $\{H(u): u \in U\}$.

$\mathfrak{R}_{1}$ uses a combination of the Fraenkel-Mostowski and Cohen methods. Let $U$ be an infinite set of urelemente and $F$ a generic choice function for domain $F=\left\{A \subset U: 2 \leqslant|A|<\aleph_{0}\right\}$. (In fact for $\omega=\Delta_{1}$ it would be enough to ensure that $\mathrm{AC}_{n}$ holds in $\mathfrak{N}_{4}$, some finite $n \geqslant 2$, by Theorem 3 ; however we wish to prove rather more about $\mathfrak{R}_{4}$ than what is stated in Theorem 5 (iv).) More precisely, a condition is a function $p$ from a finite subset of domain $F$ into $U$ such that $p(A) \in A$, each $A \in$ domain $p$. Conditions are partially ordered by inclusion, $\mathfrak{F}$ is a generic filter on the set of conditions, and $F=\bigcup \mathfrak{F} \cdot \mathfrak{M}[\mathfrak{F}]$ is the Cohen extension of $\mathfrak{M}$, and $\mathfrak{N}_{4}$ is the submodel of $\mathfrak{M}[\mathfrak{Z}]$ consisting of all its members which are hereditarily definable over $\mathfrak{M} \cup U \cup\left\{\boldsymbol{H}^{\prime}\right\}$. For this approach to forcing, see Shoenfield [16], and for ordinal definability, see Myhill and Scott [12].

For $\Re_{5}, U=\left\{u_{i}: i \in \omega\right\}$. $G$ is the group of permutations of $U$ which only move finitely many points, and $\widetilde{F}$ is generated by $\{H(u): u \in U\}$.

The restrictions given to us by $\S 2$ and $\S 3$ are these:

If $\omega \neq \Delta_{1}$, then $\Delta_{1} \neq \Delta_{2}$ (Theorem $1,(v)$ and (vi)).

If $\omega \neq \Delta$, then $\Delta_{5} \neq \Delta$ (Lemma 6$)$.

If $\omega \neq \Delta_{3}$, then $\Delta_{2} \neq \Delta_{3}$ (Lemma 6 ).

If $\Delta_{3}=\Delta$, then $\Delta_{2}=\Delta_{4}$ (corollary to Lemma 7 ).

It is easily checked that these allow only twenty-three combinations of equality and inequality in Lemma 3. We make no attempt here to discuss the relationship between $\Delta_{3}$ and $\Delta_{4}, \Delta_{5}$, though Lemmas 6 and 7 give a partial answer.
These restrictions leave us with only the following to prove in Theorem 5:

$$
\begin{aligned}
& \mathfrak{N}_{1} \vDash \omega=\Delta_{4} \neq \Delta_{5}, \Delta_{2}=\Delta_{3}, \\
& \mathfrak{R}_{2} \vDash \omega=\Delta_{4} \neq \Delta_{5}, \Delta_{3}=\Delta, \\
& \mathfrak{N}_{3} \vDash \omega=\Delta_{3} \neq \Delta_{4}=\Delta_{5}, \\
& \mathfrak{N}_{4} \vDash \omega=\Delta_{1} \neq \Delta_{2}, \Delta_{4} \neq \Delta_{5}, \Delta_{3}=\Delta, \\
& \mathfrak{N}_{5} \vDash \omega \neq \Delta_{1}, \Delta_{4} \neq \Delta_{5}, \Delta_{3}=\Delta .
\end{aligned}
$$

When the ordering principle holds, we clearly have $\omega=\Delta_{3}$. (See Levy [8] Theorem 3.) Hence $\omega=\Delta_{3}$ holds in $\mathfrak{N}_{1}$ and $\mathfrak{N}_{3}$. Levy proves that $\omega \neq \Delta_{4}$ in $\mathfrak{N}_{3}$, [8], Theorem 4 .

It is known that in $\mathfrak{R}_{1}$ any set can be put in 1-1 correspondence with a subset of $e(b) \times x$, some ordinal $\varkappa$, and hence with a set of sets of ordinals (Cohen [1], page 139). So if $X \in \mathfrak{N}_{1}$ is infinite, $X$ has a countable partition (which is in fact effectively determined by this 1-1 correspondence). This shows that $\omega=\Delta_{4}$ in $\mathfrak{N}_{1}$.

We show now that $\Delta_{4} \neq \Delta_{5}$ in $\mathfrak{N}_{1}$. Suppose $|b| \notin \Delta_{5}$. Then there is a function $f$ in $\mathfrak{N}_{1}$ from $b$ onto $b \cup\{\varnothing\}$. Let $p$ be a condition in $\widetilde{F}$ forcing " $f$ is a function". $f$ must move infinitely many points, so there are $i, j$ such that $f\left(a_{i}\right)=a_{j}, i \neq j$, and $a_{j}$ is not involved in the definition of $f$.

Let $q$ be a condition (in the generic filter) extending $p$ and forcing $f\left(a_{i}\right)=a_{j}$. Then there is a permutation $\sigma$ of $\omega$ such that $\sigma$ fixes each $k$ such that $k=i$ or $a_{k}$ is involved in the definition of $f$, but not $j$, and such that $q$ is compatible with $\sigma q$. So $q \cup \sigma q$ forces $f\left(a_{i}\right)=a_{j}$ and $f\left(a_{i}\right)$ $=a_{\sigma j}$, and this contradicts $q$ forces $f$ a function.

Hence $|b| \in \Delta_{5}-\Delta_{4}$ in $\mathfrak{N}_{1}$, so $\mathfrak{N}_{1} \vDash \Delta_{4} \neq \Delta_{5}$.

Now to show the same for $\mathfrak{R}_{2}$. Certainly $|U| \notin \Delta_{4}$. Suppose that $|U| \notin \Delta_{5}$. Then by Lemma 8 , there is a function $f$ from $U$ onto $U \cup\{\varnothing\}$, a finite $n$ such that $H(f) \supset K\left(\nabla_{n}\right)$ where $\nabla_{n}=\left\{u_{i j}: i \leqslant n\right\}$, and $i, j, k, l$ with $i, k>n, i \neq k$ and $f\left(u_{i j}\right)=u_{k l}$. Let $\sigma$ be the permutation of $U$ interchanging just $u_{k 0}$ and $u_{k 1}$. Then $\sigma \in K\left(\nabla_{n}\right)$ as $k>n$, so of $=f$, and $\sigma u_{i j}=u_{i j}$ as $i \neq k$.

Therefore $\sigma u_{k l}=\sigma f\left(u_{i j}\right)=f\left(\sigma u_{i j}\right)=f\left(u_{i j}\right)=u_{k l}$, a contradiction. So $|U| \epsilon \Delta_{5}-\Delta_{4}$ and $\mathfrak{R}_{2} \vDash \Delta_{4} \neq \Delta_{5}$.

Now let $X \in \Re_{2}$ be infinite. Let $f(\xi)=$ the least $n \in \omega$ such that $H(\xi)$ $\supset K\left(\nabla_{n}\right)$, for $\xi \in X$. If the image of $f$ is finite, let $<$ be a well-ordering of $X$ in $\mathfrak{M}$, and $n$ an upper bound for $f(\xi), \xi \in X$. Then if $\sigma \in K\left(\nabla_{n}\right), \sigma$ fixes each $\xi \in X$, and hence the well-ordering of $X$. Therefore $\kappa_{0} \leqslant|X|$.

If the image of $f$ is infinite, to show that $\kappa_{0} \leqslant{ }^{*}|X|$ it is enough to show that $f \in \mathfrak{N}_{2}$. But this follows from the fact that $H(\xi) \supset K\left(\nabla_{n}\right)$ if and only if $H(\sigma \xi) \supset K\left(V_{n}\right)$, any $\sigma$. (We omit the simple proof.)

Hence $\mathfrak{R}_{2} \vDash \omega=\Delta_{4}$. 
Now we show that in $\mathfrak{R}_{2}$ and $\mathfrak{N}_{5}, \Delta=\Delta_{3}$. In fact we show rather more, namely that any ordered set can be well-ordered. (This also holds

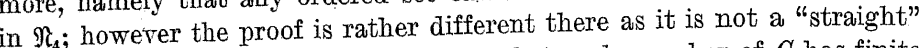
FM model). The important point here is that each member of $G$ has finite order (for $\mathfrak{N}$, this is 1 or 2 ; for $\mathfrak{N}_{5}$ this is one reason why we took only permutations of $U$ moving finitely many points). "Any ordered set can be well-ordered" implies the axiom of choice in ZF (see Rubin and Rubin [15] page 77 ), but not in FM.

Let $(X,<)$ be an ordered set. Then for some $H \in \mathfrak{F}$,

$$
H((X,<)) \supset H \text {. }
$$

Define $\sim$ on $X$ by $\xi \sim \eta$ if for some $\sigma \epsilon H, \sigma \xi=\eta$. Let $X_{0}$ be a $\sim$-class and $\xi_{0} \in X_{0}$, and suppose that $H\left(\xi_{0}\right) \not D H$. Then there is a $\sigma \in H-H\left(\xi_{0}\right)$ and as $\sigma \xi_{0} \neq \xi_{0}, \sigma \xi_{0}<\xi_{0}$ or $\xi_{0}<\sigma \xi_{0}$. As every member of $G$ has finite order, $\sigma^{n}=$ identity, some $n \epsilon \omega$. Also $\sigma \epsilon H$, so $\sigma$ preserves $<$. Therefore

or

$$
\xi_{0}>\sigma \xi_{0}>\sigma^{2} \xi_{0}>\ldots>\sigma^{n} \xi_{0}=\xi_{0}
$$

$$
\xi_{0}<\sigma \xi_{0}<\sigma^{2} \xi_{0}<\ldots<\sigma^{n} \xi_{0}=\xi_{0},
$$

each of which is impossible.

Therefore $H\left(\xi_{0}\right) \supset H$. Now by definition of $\sim, X_{0}=\left\{\sigma \xi_{0}: \sigma \in H\right\}$. But $\sigma \xi_{0}=\xi_{0}$, all $\sigma \in H$. Therefore $\left|X_{0}\right|=1$. Thus each $\sim$-class has just one member. Let $<^{\prime}$ well-order $X$ in $\mathfrak{M}$. Then every member of $H$ preserves every member of $X$, so also $<^{\prime}$. Thus $X$ can be well-ordered in $\mathfrak{N}_{2}$ (or $\mathfrak{N}_{5}$ ).

Now we move on to $\mathfrak{N}_{4}$. By Gauntt [3] Lemma 4 we have that for each $X \in \mathfrak{N}_{4}$ there is a unique minimal finite $s(X) \subset U$ such that $X$ is ordinal definable over $\mathfrak{M} \cup s(X) \cup\{F\}$ (and is contained in any other such subset of $U$ ). The function $s$ is definable in $\mathfrak{N}_{4}$, and moreoter for any finite $A \subset U$, the class of all $X \in \mathfrak{N}_{4}$ such that $s(X)=A$ has a natural well-ordering, $w(A)$, say, in $\mathfrak{N}_{4}$, given by the minimal triple $(\Phi, \alpha, i)$, where $\Phi$ is a formula defining $X$ in terms of the ath member of $\mathfrak{M}$ and $F$ (if $i=0$ ), or not with $F$ (if $i=1$ ), and $A$.

We are now able to show that $\mathfrak{N}_{4}$ satisfies $W$ : any set of non-empty well-orderable sets has a choice function. A similar proof shows that it holds in $\mathfrak{N}_{1}$ and $\mathfrak{N}_{3}$ (see Läuchli [6] pages 33,35 ), but we do not need that here.

Let $X$ be a set of non-empty well-orderable sets in $\mathfrak{N}_{4}$, and let $Y \in X$. As $Y$ can be well-ordered in $\mathfrak{N}_{4}$, there is a 1-1 map in $\mathfrak{N}_{4}$ from $Y$ into $O n$ (= the class of all ordinals). Hence for some finite $A \subset U$, every member of $Y$ is ordinal definable over $\mathfrak{M} \cup A \cup\{F\}$. Therefore $\cup\{s(\xi): \xi \in Y\}$ is finite. Let it be $A$. Then $F$ defines an ordering of $P(A)$. Let $B$ be the first member of $P(A)$ in this ordering such that for some $\xi \in Y, s(\xi)=B$, and let $g(Y)$ be the first member of $Y \cap\{Z: s(Z)=B\}$ in $w(B) . g$ is defined in terms of $F$, and so lies in $\mathfrak{R}_{4}$.

$W$ implies as a very special case $\mathrm{AC}_{<\infty}$ (or indeed $\mathrm{AC}_{2}$ etc.) and by the corollary to Theorem $3, \omega=\Delta_{1}$ in $\mathfrak{N}_{4}$.

Now to show that $\omega \neq \Delta_{2}$ in $\mathfrak{N}_{4}$ we show that $|\dot{U}| \epsilon \Delta_{2}$. Let $(\nabla,<)$ be an infinite ordered partition of $U$ in $\mathfrak{R}_{4}$. Then for some condition $p$ in $\mathfrak{F}$, $p$ forces " $<$ is a linear ordering". Let $A$ be the set of all wrelemente involved in $p$ and in $s((V,<))$. Define $\sim$ on $U-A$ by $u \sim v$ if whenever $\varnothing \neq B \subset A, F(B \cup\{u\})=u$ and $F^{\prime}(B \cup\{v\})=v$ or $F^{\prime}(B \cup\{u\})=F^{\prime}(B \cup\{v\})$.

$\sim$ is an equivalence relation and there are only finitely many equivalence classes. Since $V$ is infinite, there are $u_{1}, u_{2} \in U-A$, such that the $\sim$ - class of $u_{1}$ is infinite, $u_{1} \sim u_{2}$, and if $u_{1} \in v_{1} \in V, u_{2} \in v_{2} \in V, v_{1}<v_{2}$. (In fact every $\sim$-class is infinite but we do not need that here.)

Let $q \geqslant p, q \in \mathfrak{F}, q$ force $v_{1}<v_{2}$. Let $B$ be finite, $B \cap A=\emptyset$, and be such that every member of $U$ occurring in $q$ or $s\left(v_{1}\right), s\left(v_{2}\right), s\left(u_{1}\right), s\left(u_{2}\right)$ is in $A \cup B$, and $u_{1}, u_{2} \in B$.

Let $B=\left\{u_{1}, u_{2}, w_{1}, w_{2}, \ldots, w_{n}\right\}$. Since the $\sim$-class of $u_{1}$ is infinite, there is a $u_{3} \in U-(A \cup B)$ such that $u_{1} \sim u_{3}$. Let $s_{1}, s_{2}, \ldots, s_{n}, t_{1}, t_{2}, \ldots, t_{n}$ be distinct members of $U-\left(A \cup B \cup\left\{u_{3}\right\}\right)$.

Let

$$
B_{0}=\left\{u_{2}, u_{3}, s_{1}, s_{2}, \ldots, s_{n}\right\} \quad \text { and } \quad B_{1}=\left\{u_{3}, u_{1}, t_{1}, t_{2}, \ldots, t_{n}\right\} .
$$

$\sigma, \tau$, permutations of $U$. are defined thus:

$$
\begin{aligned}
& \sigma u_{1}=u_{2}, \sigma u_{2}=u_{3}, \sigma u_{3}=u_{1}, \sigma w_{i}=s_{i}, \sigma s_{i}=w_{i}, \\
& \tau u_{1}=u_{3}, \tau u_{3}=u_{2}, \tau u_{2}=u_{1}, \tau w_{i}=t_{i}, \tau t_{i}=w_{i},
\end{aligned}
$$

and $\sigma$ and $\tau$ are the identity on points not mentioned.

We observe that $\sigma B=B_{0}, \tau B=B_{1}$, and $\sigma, \tau$ both fix $A$ pointwise.

We wish to show that $q \cup \sigma q \cup \tau q$ is a condition. For this we show that if $X \in$ domain $q$ ndomain $\sigma q, q(X)=(\sigma q)(X)$, and similarly for $q, \tau q$ and $\sigma q, \tau q$.

By the choice of $A$ and $B$, domain $q \subset P(A \cup B)$. Hence domain $\sigma q \subset P\left(A \cup B_{0}\right)$ and domain $\tau q \subset P\left(A \cup B_{1}\right)$.

Thus if $X \in$ domain $q \cap$ domain $\sigma q$,

$$
X \subset(A \cup B) \cap\left(A \cup B_{0}\right)=A \cup\left\{u_{2}\right\} \text {. }
$$

If $q(X)=u_{2}, \quad F(X)=u_{2}$ as $q \subset F$, so $F\left((X \cap A) \cup\left\{u_{1}\right\}\right)=u_{1}$ as $u_{1} \sim u_{2}$. Therefore

$$
\left((X \cap A) \cup\left\{u_{1}\right\}, u_{1}\right) \in F \quad \text { and } \quad\left((X \cap A) \cup\left\{u_{2}\right\}, u_{2}\right) \in \sigma F \text {, }
$$

as

$$
\sigma(X \cap A)=X \cap A \quad \text { and } \quad \sigma u_{1}=u_{2} .
$$


This just says that $\left(X, u_{2}\right) \in \sigma F$. Since $\sigma q \subset \sigma F, X \in$ domain $\sigma q$, and $\sigma F$ is a function,

$$
(\sigma q)(X)=(\sigma F)(X)=u_{2}=q(X), \text { as desired. }
$$

Similarly if $q(X) \in A$.

Hence $q \cup \sigma q$ is a condition. In a similar way we get that $q \cup \tau q$, $\sigma q \cup \tau q$ are conditions, and hence that $q \cup \sigma q \cup \tau q$ is a condition.

Since $q$ forces $v_{1}<v_{2}$, we have $\sigma q$ forces $v_{2}<v_{3}$ and $\tau q$ forces $v_{3}<v_{1}$ where $u_{3} \in v_{3} \in \nabla$, because $\sigma, \tau$ fix $A$ and hence $<$ and $V$. Therefore $q \cup \sigma q \cup \tau q$ forces $v_{1}<v_{2}$ and $v_{2}<v_{3}$ and $v_{3}<v_{1}$ which contradicts $q \geqslant p$ and $p$ forces $<$ to be a linear ordering.

Hence $U$ has no infinite ordered partition in $\mathfrak{R}_{4}$, and so $|U| \epsilon \Delta_{2}-\omega$. We have now incidentally proved the following, which is a strengthening of Läuchli [6].

THEOREM 6. If $\mathrm{ZF}$ is consistent, then so is $\mathrm{ZF}+\mathrm{W}$ : any set of nonempty well-orderable sets has a choice function + there is an infinite set with no infinite ordered partition.

To obtain the ZF consistency result as stated here from our proof using urelemente, we use Pincus [14], 4B2. Alternatively a direct proof is not hard. One adds an infinite set of infinite sets of generic reals. (See Gauntt [3].)

We are now left with the following to prove from Theorem 5 .

$$
\begin{aligned}
& \mathfrak{N}_{3} \vDash \Delta_{4}=\Delta_{5}, \\
& \mathfrak{N}_{4} \vDash \Delta_{4} \neq \Delta_{5}, \Delta_{3}=\Delta, \\
& \mathfrak{N}_{5} \vDash \omega \neq \Delta_{1}, \Delta_{4} \neq \Delta_{5} .
\end{aligned}
$$

Firstly to show that $\Delta_{3}=\Delta$ in $\mathfrak{N}_{4}$. Let $(X,<)$ be an infinite ordered set in $\mathfrak{R}_{4}$. If for some finite subset $A$ of $U,\{\xi \in X: s(\xi)=A\}$ is infinite, this provides a countable subset of $X$ by $w(A)$. If for each finite $A \subset U,\{\xi \in X: s(\xi)=A\}$ is finite, then by $W$ in $\mathfrak{N}_{4}$ we may suppose that each $|\{\xi \in X: s(\xi)=A\}|$ is $\leqslant 1$ (replacing $X$ by an infinite subset). This amounts to supposing $X \subset e(U)$. But by Theorem 4 and $|U| \in \Delta_{2}, X$ cannot be ordered in $\mathfrak{N}_{4}$, a contradiction.

Now suppose that $\nabla$ is an infinite subset of $U$ in $\mathfrak{N}_{5}$. Then for some finite $A \subset U, H(V) \supset K(A)$. As $V$ is infinite, there is some $u_{i} \in V-A$. Let $u_{j}$ be any other member of $U-A$. Then $\sigma$, the permutation of $U$ which interchanges $u_{i}$ and $u_{j}$, lies in $K(A)$, so $\sigma V=V$. Therefore $u_{j} \in V$. Thus $U-V$ is finite. This shows that $U$ is not the disjoint union of two infinite sets in $\mathfrak{N}_{5}$, i.e. that $|U| \epsilon \Delta_{1}-\omega$. Therefore $\omega \neq \Delta_{1}$ in $\mathfrak{N}_{5}$.

We next show that $|e(U)| \in \Delta_{5}-\Delta_{4}$ in $\mathfrak{N}_{5}$. Certainly $e(U)$ has a countable partition, as $e(U)=\bigcup\left\{[U]^{n}: n \in \omega\right\}$. Suppose that $|e(U)| \notin \Delta_{5}$. Then by Lemma 8 there is a map $f$ from $e(U)$ onto $e(U) \cup\{1\}(1 \notin e(U)\}$ and an increasing function $n: \omega \rightarrow \omega$ such that for each $i, f^{-(i+1)}(\{1\})$ $C[U]^{n(i)}$.

As $f \in \mathfrak{N}_{5}$, for some finite $A \subset U, H(f) \supset K(A)$. For some $i, n(i)$ $>|A|$. So there is an $X \in e(U)$ satisfying $X \not \subset A$ and $X \in \cup\left\{f^{-(i+1)}(\{1\})\right.$ : $i \in \omega\}$, and with respect to this, $|X \cap A|$ is maximal. (We may assume maximality because $A$ is finite.)

Let $f(Y)=X$. Then as $n$ is an increasing function,

$$
|Y|=n(i+1)>n(i)=|X| \text {. }
$$

Case I. $X-(Y \cup A) \neq \varnothing$. Let $u \in X-(Y \cup A)$. Let $u^{\prime}$ be a member of $U-(X \cup Y \cup A)$. $\left(X \cup Y \cup A\right.$ is finite.) Then the permutation $\left(u u^{\prime}\right)$ is in $K(A)$, and hence also in $H(f)$.

Since $(Y, X) \in f,(\sigma Y, \sigma X) \in f$, where $\sigma$ is this permutation. But $\sigma Y=Y$ as neither $u$ nor $u^{\prime}$ is in $Y$, and $\sigma X \neq X$ as $u \in X$ but $u^{\prime} \notin X$. This contradicts $f$ a function.

Case II. $X \subset Y \cup A$. Then $X-A \subset Y-A$. By the maximality of $|X \cap A|,|X \cap A| \geqslant|Y \cap A|$. Therefore

$$
|X-A|=|X|-|X \cap A| \leqslant|X|-|Y \cap A|<|Y|-|Y \cap A|=|Y-A| .
$$

Since $X \not \subset A$, we get

$$
\emptyset \neq X-A \subset Y-A \text { and } X-A \neq Y-A .
$$

Let $u \in X-A, u^{\prime} \in(Y-A)-X$, and let $\sigma=\left(u u^{\prime}\right)$. Then $u$ and $u^{\prime}$ are both in $Y$, but $u \in X$ and $u^{\prime} \notin X$.

As in case $I$ this gives a contradiction.

Therefore $\Delta_{4} \neq \Delta_{5}$ in $\mathfrak{N}_{5}$.

There remain now to prove, $\Delta_{4}=\Delta_{5}$ in $\mathfrak{\Re}_{3}$ and $\Delta_{4} \neq A_{5}$ in $\mathfrak{R}_{4}$. Let $|X| \notin \Delta_{4}$ in $\mathfrak{R}_{3}$. Then $X=\bigcup\left\{X_{i}: i \in \omega\right\}, X_{i}$ disjoint, and the sequence $\left\{\left(X_{i}, i\right): i \in \omega\right\}$ in $\mathfrak{R}_{3}$. This means that for some finite $A \subset U, H\left(X_{i}\right) \supset K(A)$, each $i \in \omega$.

$\sim$ is defined on $X_{i}$ by $\xi \sim \eta$ if for some $\sigma \epsilon K(A), \sigma \xi=\eta$. Let $\emptyset$ $\neq Y_{i} \subset X_{i}, Y_{i}$ a $\sim$-class, each $i \in \omega$. Then $H\left(Y_{i}\right) \supset K(A)$, each $i$, so $\bigcup\left\{Y_{i}: i \in \omega\right\} \in \mathfrak{R}_{3}$. This amounts to supposing, without loss of generality, that each $X_{i}$ is a $\sim$-class. Let $\xi_{i} \in X_{i}$. Then by Mostowski [11] page 239 we know that there is a unique $B_{i}, B_{i}$ finite and contained in $U, B_{i} \cap$ $\cap A=\varnothing$, such that $H\left(\xi_{i}\right) \cap K(A)=K\left(B_{i} \cup A\right)$, each $i \in \omega$. Let $I_{0}, I_{1}, \ldots, I_{n}$ be the open intervals of $U$ under $A$. Then if $\eta_{i} \subset U-A$ and $\left|\eta_{i} \cap I_{j}\right|$ $=\left|B_{i} \cap I_{j}\right|$, each $j \leqslant n$, there is a $\sigma \in K(A)$ mapping $B_{i} 1$-1 onto $\eta_{i}$.

Therefore if we map $X_{i}=\left\{\sigma \xi_{i}: \sigma \in K(A)\right\}$ to $\left\{\sigma B_{i}: \sigma \in K(A)\right\}$ by $\sigma \xi_{i} \rightarrow \sigma B_{i}$ the map is $1-1$ and "onto", because $H\left(\xi_{i}\right) \cap K(A)=K\left(B_{i} \cup A\right)$, it lies in $\mathfrak{R}_{3}$ (easily checked), and its image is

$$
\left\{\eta_{i} \subset U-A:\left|\eta_{i} \cap I_{j}\right|=\left|B_{i} \cap I_{j}\right| \text {, each } j \leqslant n\right\} .
$$


Thus we may suppose that $X_{i}$ is of the form

$$
\left\{\eta_{i} \subset U-A:\left|\eta_{i} \cap I_{j}\right|=m_{j}(i)\right\}
$$

for some $(n+1)$-tuple $\left(m_{0}(i), m_{1}(i), \ldots, m_{n}(i)\right)$.

By discarding some of the $X_{i}$ 's, (but retaining infinitely many) we may suppose that $i \leqslant j \rightarrow m_{0}(i) \leqslant m_{0}(j)$. (Consider the two cases $m_{0}(i)$ bounded and unbounded), and similarly for the other co-ordinates one by one. Thus we may suppose that for each $k \leqslant n, i \leqslant j \rightarrow m_{k}(i)$ $\leqslant m_{k}(j)$.

We are now able to map $X$ onto $X \cup\{1\}(1 \notin X)$.

If $\eta_{i} \in X_{i}, i=0$, let $f\left(\eta_{i}\right)=1$. If $\eta_{i} \in X_{i}, i>0$, let $f\left(\eta_{i}\right)=\zeta_{i}$ where $\zeta_{i} \subset U-A$ and for each $j \leqslant n, \zeta_{i} \cap I_{j}$ are the first $m_{j}(i-1)$ members of $\eta_{i} \cap I_{j}$ in the ordering of $U$.

It is then easily checked that $f \in \mathfrak{N}_{3}$ and $f$ is "onto", as desired. Hence $\Delta_{4}=\Delta_{5}$ in $\mathfrak{N}_{3}$.

In fact by pushing this proof a little further one can show that in $\mathfrak{N}_{3}$ any cardinal $x$ can be written as $y+z$, where $y \in \Delta_{4}$, and $2 z \leqslant^{*} z$.

Finally we show that in $\mathfrak{R}_{4}, \Delta_{4} \neq \Delta_{5}$. Let $u \in U$, and for each $n \in \omega$,

$X_{n}=\left\{\xi \in[U-\{u\}]^{n}: F(\{u\} \cup \xi) \epsilon \xi\right.$, and if $\emptyset \neq A \neq \xi, A \subset \xi$, then

$$
F(\{u\} \cup A)=u\} \text {. }
$$

Each $X_{n}$ is non-empty becanse no condition can force it to be empty. Thus if $X=\bigcup\left\{X_{n}: n \in \omega\right\}, \mathrm{s}_{0} \leqslant^{*}|X|$.

We show that $|X| \in \Delta_{5}$ in $\mathfrak{N}_{4}$. If not there is a function $f: X \rightarrow X \cup\{1\}$ $(1 \notin X)$, "onto", as given by Lemma 8. (Each $\left|X_{n}\right| \in \Delta_{4}$ by Theorem 1 (vi).)

Then for some $p \in \mathfrak{F}, p$ forces " $f$ is a function". Let $A$ be a finite subset of $U$ containing $u, s(f)$, and any members of $U$ involved in $p$. By choice of $f$ there are $\xi, \eta \subset U$ such that $0<|\eta|<|\xi|,(\xi, \eta) \in f^{n}$, some $n>0, \eta \cap A=\xi \cap A$.

Let $q \in \widetilde{F}, q \geqslant p$, and $q$ force $(\xi, \eta) \in f^{n}$. If $\eta \subset \xi$, by definition of $X_{n}$, and since $\emptyset \neq \eta \neq \xi, F(\{u\} \cup \eta)=u$. However this is contrary to $\eta \in X$. Therefore $\eta \not \subset \xi$.

Let $v \in \eta-\xi$, and let $B$ be the set of all members of $U$ involved in $A, \xi, \eta, q$; let $w \in U-B$. Since $\eta \cap A=\xi \cap A, v \notin A$.

If $\sigma$ is the permutation of $U$ interchanging $v$ and $w$, then

$$
\sigma q \text { forces }(\sigma \xi, \sigma \eta) \in \sigma f^{n} \text {. }
$$

But $\sigma$ fixes $s(f), \xi$ pointwise, so

$$
\sigma q \text { forces }(\xi, \sigma \eta) \epsilon f^{n} \text {. }
$$

It is easily seen that $q$ and $\sigma q$ are compatible.
Hence $q \cup \sigma q$ forces $(\xi, \eta) \in f^{n}$ and $(\xi, \sigma \eta) \in f^{n}$, and this contradicts $\eta \neq \sigma \eta, q \geqslant p$, and $p$ forces " $f$ is a function".

We conclude by answering a question of Tarski's as to whether $|\Delta|=2^{\text {NoO }}$ is possible. (See [17].)

THEOREM 7. Each of $\mathfrak{N}_{1}, \mathfrak{N}_{2}$, and $\mathfrak{N}_{5}$ (of Theorem 5) satisfies $|\Delta|=2^{N_{0}}$ and there is a set of $2^{\mathrm{N}_{0}}$ mutually incomparable members of $\Delta$.

CoRollary. If ZF is consistent, so are $\mathrm{ZF}+|\Delta|=2^{\mathrm{N}_{0}}+2^{\mathrm{N}_{0}}$ is a wellordered cardinal + there is a set of $2^{\text {No }_{0}}$ mutually incomparable members of $t$, and $\mathrm{ZF}+|\Delta|=2^{\mathrm{K}_{0}}+2^{\mathrm{No}}$ is not a well-ordered cardinal + there is a set of $2^{\text {No }}$ mutually incomparable members of $\Delta$.

Proof. The first part of the corollary follows from the fact that $\mathfrak{N}_{2}$ (or $\mathfrak{R}_{5}$ ) satisfies the desired statement (remember that in any FraenkelMostowski model, the real numbers can be well-ordered), by use of Pincus [14] 2B7. (This fact was pointed out to us by Dr Pincus).

Firstly we show that $|\Delta| \leqslant 2^{\mathbb{N}_{n}}$ in $\mathfrak{R}_{1}$. Let $\mathfrak{M}^{\prime}$ be the extension of $\mathfrak{M}$ formed by adding a sequence $\left\{\left(a_{i}, i\right): i \in \omega\right)$ of generic reals to $\mathfrak{M}$, where we suppose that $\mathfrak{M} \vDash C H$. (i.e. $2^{\aleph_{0}}=\kappa_{1}$ ). Cohen shows on page 125 of [1] that $\mathfrak{M}^{\prime} \vDash C H$. $\mathfrak{N}_{1}$ may be viewed as a submodel of $\mathfrak{M}^{\prime}$. In fact $\xi \in \mathfrak{N}_{1} \leftrightarrow \xi \subset \mathfrak{N}_{1}$ and $\xi$ is $\mathfrak{M}$-definable over $\left\{a_{i}: \quad i \in \omega\right\} \cup\{b\}$ where $b=\left\{a_{i}: i \in \omega\right\}$.

Since $\omega_{1}$ and $\omega_{2}$ are unchanged in the extension $\mathfrak{M} \rightarrow \mathfrak{M}^{\prime}$ (see Cohen [1], pages 131-132), they are the same in $\mathfrak{N}_{1}$ too.

Now any set in $\mathfrak{N}_{1}$ can be put into 1-1 correspondence in $\mathfrak{N}_{1}$ with a subset of $e(b) \times *$, some ordinal * (by Cohen [1] page 139; remember that $e(b)$ is the set of finite subsets of $b)$. Hence any Dedekind finite set in $\mathfrak{N}_{1}$ can be put into 1-1 correspondence with a subset of $e(b) \times \omega$. (This shows by the way, that $\Delta$ is a set; in general it may be a proper class.) So $|\Delta| \leqslant 2^{*} 2^{|e(b) \times \omega|}$.

Suppose that $s_{2} \leqslant|\Delta|$ in $\mathfrak{R}_{1}$. Then $s_{2} \leqslant 2^{|e(b) \times \omega|}$. However $\mathfrak{N}_{1} \subset \mathfrak{M} \mathfrak{N}^{\prime}$, $\omega_{2}^{\mathfrak{M}_{1}}=\omega_{2}^{\mathfrak{M}^{\prime}}$, and $|e(b) \times \omega|=\aleph_{0}$ in $\mathfrak{M}^{\prime}$. So this violates $\mathrm{CH}$ in $\mathfrak{M}^{\prime}$.

Therefore $\kappa_{2} \nless|\Delta|$ in $\mathfrak{N}_{1}$.

Since $\Delta$ is a set there is a 1-1 mapping $f$ from $\Delta$ into $P(\omega) \times x$, some ordinal $\%$. We may suppose that for each $A \in P(\omega), f^{\prime \prime} \Delta \cap(\{A\} \times \%)$ is an initial segment of $\{A\} \times \%$. Let its order-type be $\alpha_{A}$ :

Then, as $f$ is $1-1, \alpha_{A}$ can be mapped 1-1 into $\Delta$. Since $s_{2} \nless|\Delta|$, $\alpha_{A}<\omega_{2}$. The same argument that was used to show that $s_{2} \nless|\Delta|$ in $\mathfrak{N}_{1}$ shows that $s_{2} \star^{*} 2^{\boldsymbol{N}_{0}}$ in $\mathfrak{N}_{1}$. Hence the values of $\alpha_{A}$ for $A \in P(\omega)$ are bounded below $\omega_{2}$.

This means that $|\Delta| \leqslant 2^{\aleph_{0}} \cdot s_{1}=2^{\aleph_{0}}$. (Because $s_{1} \leqslant 2^{\aleph_{0}}$ holds in $\Re_{1}$ )

Now let $\mathcal{A}$ be a set of $2^{\text {No }}$ "almost disjoint" infinite subsets of $\omega$ in $\mathfrak{N}_{1}$, i.e. $A_{1}, A_{2} \in \mathcal{A}, A_{1} \neq A_{2} \rightarrow A_{1} \cap A_{2}$ finite. For $A \in \mathfrak{A}$, let $b_{A}=\{a \in b$ : 
$A \subset a\}$. Then $\left\{\left|b_{A}\right|: A \in \mathcal{A}\right\}$ is a set of $2^{\mathrm{x}_{0}}$ mutually incomparable members of $\Delta$. The details are omitted.

We now turn to $\mathfrak{R}_{2}$ and $\mathfrak{R}_{5}$. In each case every member of the filter $\mathfrak{\&}$ has finite index in $G$, so $\mathfrak{F}$ is countable. Let $\mathfrak{F}=\left\{G_{0}, G_{1}, G_{2}, \ldots\right\}$.

For $\mathfrak{N}_{2}$, let $v_{n}=\left(u_{00}, u_{01}, u_{10}, u_{11}, \ldots, u_{n 0}, u_{n 1}\right)$ and for $\mathfrak{R}_{5}$ let $v_{n}$ $=\left(u_{0}, u_{1}, u_{2}, \ldots, u_{n-1}\right)$.

Then for each $H \in \widetilde{F}, H \supset H\left(v_{n}\right)$, some $n \in \omega$. Let $n_{i}=$ the least $n$ such that $G_{i} \supset H\left(v_{n}\right)$.

Let

$$
w_{i}=\left\{\sigma \boldsymbol{v}_{n i}: \sigma \in G_{i}\right\} \text { and } X_{n i}=\left\{\sigma w_{i}: \sigma \in H\left(\boldsymbol{v}_{n}\right)\right\} .
$$

Firstly $H\left(w_{i}\right)=G_{i}$. For certainly $G_{i} \subset H\left(w_{i}\right)$. Conversely, if $\sigma \in H\left(w_{i}\right)$, $\boldsymbol{v}_{m_{i}} \in w_{i}$ shows that $\sigma v_{n_{i}} \in w_{i}$. That is, for some $\tau \in G_{i}, \sigma v_{m i}=\tau v_{m i}$. This gives $\tau^{-1} \sigma \in H\left(v_{m_{i}}\right)$, and as $H\left(v_{m_{i}}\right) \subset G_{i}$, by definition of $n_{i}, \sigma \in \tau G_{i}=G_{i}$. This shows that $H\left(w_{i}\right)=G_{i}$, as desired.

Now let $X \in \mathfrak{R}_{2}\left(\mathfrak{R}_{5}\right.$ respectively), $|X| \in \Delta$. Then for some $n, H(X)$ $\supset H\left(\boldsymbol{v}_{n}\right)$. Let $\mathfrak{X}$ be the set of $H\left(\boldsymbol{v}_{n}\right)$-orbits of $X$, and let $\mathfrak{X}=\left\{X_{a}: a<x\right\}$ be a well-ordering of $\mathfrak{X}$ in $\mathfrak{M}$. Since $H\left(X_{a}\right) \supset H\left(\boldsymbol{y}_{n}\right)$, each $\alpha$, this well-ordering is also in $\mathfrak{N}_{2}$ (or $\mathfrak{N}_{5}$ ). Pick $\xi_{a} \in X_{a}$, each $\alpha$ (choice in $\mathfrak{M}$ ), and for each $\alpha$ let $i_{\alpha}$ be the unique $i \in \omega$ such that $H\left(\xi_{a}\right) \cap H\left(\boldsymbol{v}_{n}\right)=G_{i}$.

Let $A_{i}=\left\{\alpha<x: i_{a}=i\right\}$. We define the map $f: X \rightarrow \bigcup\left\{x_{i} \times X_{n i}^{\prime}: i \in \omega\right\}$ thus, where $\varkappa_{i}=\left|A_{i}\right|$ ( $x_{i}$ an initial ordinal, as $A_{i}$ is a set of ordinals, so can be well-ordered), where $X_{n i}^{\prime}=X_{n i} \times\{i\}$; this ensures disjointness of the $X_{n i}^{\prime}$.

Let $f_{i}$ map $A_{i} 1-1$ onto $\varkappa_{i}$.

If $\xi \in X, \xi \in \bar{X}_{a}$, for a unique ordinal $\alpha<x$, since $\mathfrak{X}$ is a partition of $X$. As $X_{a}$ is an $H\left(v_{n}\right)$-orbit, there is a $\sigma \in H\left(v_{n}\right)$ such that $\xi=\sigma \xi_{a}$. We let $f(\xi)=\left(f_{i_{a}}(\alpha), \sigma w_{i_{a}}, i_{a}\right)$.

We have to check that $f$ is $1-1$, "onto", and is in $\mathfrak{R}_{2}$ (or $\mathfrak{N}_{5}$ ).

1. Well-defined and 1-1.

$$
\begin{aligned}
\sigma \xi_{a}=\tau \xi_{a}, \sigma, \tau \epsilon H\left(v_{n}\right) & \leftrightarrow \tau^{-1} \sigma \in H\left(\xi_{\alpha}\right) \cap H\left(v_{n}\right)=G_{i_{\alpha}} \\
\leftrightarrow & \leftrightarrow \tau^{-1} \sigma \in H\left(w_{i_{\alpha}}\right) \leftrightarrow \sigma w_{i_{\alpha}}=\tau w_{i_{\alpha}} .
\end{aligned}
$$

2. "Onto". This follows from the definition of $X_{n i}^{\prime}$, and the fact that each $X_{a}$ is an $H\left(v_{n}\right)$-orbit.

3. $f \in \mathfrak{N}_{2}$ (or $\mathfrak{N}_{\overline{5}}$ ). In fact $H(f) \supset H\left(\boldsymbol{v}_{n}\right)$. For if $\tau \in H\left(\boldsymbol{v}_{n}\right)$, and $\sigma \xi_{a} \in X_{a}$,

$$
\tau f\left(\sigma \xi_{a}\right)=\tau\left(f_{i_{a}}(\alpha), \sigma w_{i_{a}}, i_{\alpha}\right)=\left(f_{i_{\alpha}}(\alpha), \tau \sigma w_{i_{\alpha}}, i_{\alpha}\right)=f\left(\tau \sigma \xi_{a}\right) .
$$

Thus any set $X$ in $\mathfrak{N}_{2}\left(\mathfrak{N}_{5}\right)$ can be put in 1-1 correspondence in $\mathfrak{R}_{2}\left(\mathfrak{N}_{5}\right)$ with a set of the form $\bigcup\left\{\varkappa_{i} \times X_{n i}^{\prime}: i \in \omega\right\}$, for some $n$, and sequence $\varkappa_{i}$ of well-ordered cardinals.

Now we supposed that $X$ was Dedekind finite. Hence each $\varkappa_{i}$ is finite.
Thus if we map $\omega \times \omega^{\omega}$ by $g$, thus:

$$
g((n, f))=\left|\bigcup\left\{f(i) \times X_{n i}^{\prime}: i \epsilon \omega\right\}\right|
$$

we certainly have $\Delta \subset g^{\prime \prime}\left(\omega \times \omega^{\omega}\right)$, (and in fact $=$ ).

We now show that $g \in \mathfrak{R}_{2}$ (or $\mathfrak{R}_{5}$ ). In fact $H(g)=G$. To show this it is enough that $|\sigma X|=|X|$, any $\sigma \in G, X \in \mathfrak{N}_{2}\left(\mathfrak{N}_{5}\right)$. (This actually shows that any set of cardinals can be well-ordered.)

For $\mathfrak{N}_{2}$ we show that $H(\sigma \uparrow X) \supset H(X)$ and for $\mathfrak{N}_{5}, H(\sigma \uparrow X) \supset H(X) \cap$ $\cap H\left(v_{n}\right)$, where $n$ is the least integer such that $\sigma$ fixes all $u_{m}$ with $m \geqslant n$.

For $\tau \in H(X)\left(H(X) \cap H\left(v_{n}\right)\right.$ respectively), $\tau$ commutes with $\sigma$, so

$$
\begin{aligned}
\tau(\sigma \uparrow X) & =\tau\{(\xi, \sigma \xi): \xi \in X\}=\{(\tau \xi, \tau \sigma \xi): \xi \in X\} \\
& =\{(\tau \xi, \sigma \tau \xi): \xi \in X\} \\
& =\{(\tau \xi, \sigma \tau \xi): \tau \xi \in X\} \quad \text { as } \quad \tau \in H(X) \\
& =\{(\xi, \sigma \xi): \xi \in X\}=\sigma \uparrow X .
\end{aligned}
$$

Therefore $|\Delta| \leqslant 2^{*}{ }^{\aleph_{0}}$. Since $2^{\aleph_{0}}$ is a well-ordered cardinal, we have $|\Delta| \leqslant 2^{\boldsymbol{N}_{0}}$.

Finally to show that in $\mathfrak{N}_{2}$ and $\mathfrak{N}_{5}$ there is a set of $2^{\mathrm{N}_{0}}$ mutually incomparable members of $\Delta$, let $A$ again be a set of $2^{N_{0}}$ almost disjoint infinite subsets of $\omega$.

Then $\left\{\left|X_{A}\right|: A \in \mathscr{A}\right\}$ is a set of $2^{\mathrm{N}_{0}}$ mutually incomparable members of $\Delta$, where $X_{A}=\bigcup\left\{X_{0 i}^{\prime}: i \in A\right\}$.

\section{References}

[1] P. J. Cohen, Set Theory and the Continuum Bypothesis, Benjamin 1966.

[2] R. J. Gauntt, Axiom of Choice for finite sets, Notices of the American Mathematical Society, February 1970, p. 454 .

[3] - Axiom of Ohoice for finite sets. Unpublished.

[4] J. D. Halpern and A. Levy, The Boolean Prime Ideal Theorem does not imply the Axiom of Choice, American Mathematical Society, Proceedings of Symposia in Pure Mathematics, Vol. XIII, Part 1, pp. 83-134.

[5] T. Jech and A. Sochor, Applications of the $\theta$-model, Bulletin de l'Académie Polonaise des Sciences 14 (1966), pp. 297-303 and pp. 351-355.

[6] H. Läuchli, The independence of the ordering principle from a restricted Axiom of Choice, Fund. Math. 54 (1964), pp. 31-43.

[7] A. Levy, The Fraenkel-Mostowski method for independence proofs in set theory, Symposium on the Theory of Models, Berkeley 1963 (1965), pp. 221-228.

[8] - The independence of various definitions of finiteness, Fund. Math. 46 (1958), pp. $1-13$.

[9] A. Lindenbaum and A. Tarski, Communication sur les recherches de la théorie des ensembles, Comptes Rendus des Séances de la Société des Sciences et des Lettres de Varsovie, Cl. III, Sciences, Mathématiques et Physiques (1926), pp. 299-330.

[10] A. Mostowski, Axiom of Choice for finite sets, Fund. Math. 33 (1945), pp. 137-168. 3 - Fundamenta Mathematicae LXXXIV 
[11] A. Mostowski. Über die Unabhängigkeit des Wohlordnungssatzes vom Ordnungsprinzip, Fund. Math. 32 (1939), pp. 201-252.

[12] J. Myhill and D. Scott, Ordinal definability, American Mathematical Society, Proceedings of Symposia in Pure Mathematics, Vol. XIII, Part 1, pp. 271-278. Droceedings of Symptrins, Support structures for the Axiom of Ohoice, J. Symb. Logic 36 (1971), pp. 28-38.

[14] - Zermelo-Fraenkel consistency results by Fraenkel-Mostowski methods, J. Symb. Logic 37 (1972), pp. 721-743.

[15] H. Rubin and J. Rubin, Equivalents of the Axiom of Ohoice, 1963.

[16] J. R. Shoenfield, Unramified forcing, American Mathematical Society, Proceedings of Symposia in Pure Mathematics, Vol. XIII, Part 1, pp. 357-381.

[17] A. Tarski, On the existence of large sets of Dedelind cardinals, Notices of the American Mathematical Society, October 1965, p. 719.

[18] - Sur les ensembles finis, Fund. Math. 6 (1924), pp. 45-95.

[19] J. Truss, On successors in cardinal arithmetic, Fund. Math. 78 (1973), pp. 7-21.

MATHEMATICAL INSTITUTE

St. Giles, Oxford

Regen par la Rédaction le 10. 11. 1972

\section{О теореме Виеториса в категории гомотопий и одной проблеме Борсука}

\author{
С. Богатый (Москва)
}

Абстракт. Рассматриваются отображения (*) метризуемых компактов, у которых полные прообразы точек аптроксимативно связны в некоторой размерности $m$ [7]. В частности, доказывается аналог теорем Виеториса [23] и Смейла [22]: если для отображения $f: X \rightarrow Y$ компакта $X \in \mathrm{LC}^{m}$ на компакт $Y$ прообразы $f^{-1}(y) \in \mathrm{AC}^{m}$ для всех $y \in Y$, то $\bar{I} \in \mathrm{LC}^{m}$ ил индуцированное отображение $f_{*}:[Z, X] \rightarrow[Z, Y]$ (классов $[Z, X]$ гомотопных отображений) биективно, как только $\operatorname{dim} Z \leqslant m$. Это позволяет дать частичный ответ на одну проблему K. Борсука [5]: если для отображения $f: X \rightarrow Y$ конечномерного компакта $X$ на конечномерный компакт $Y$ прообразы $f^{-1}(y) \in \mathrm{FAR}$ для всех $y \in Y$, то $\operatorname{Sh} X=$ $=\operatorname{Sh} Y$. Кроме этого, в ответ на вопрос К. Борсука [7] доказывается, что фундаментальные абсолютные ретракты это в точности подвижные компакты, которые апшроксимативно связны во всех размерностях.

Пусть $X$ и $Y$ компактные метрические пространства и пусть отображение $f: X \rightarrow Y$ является на. Теорема Виеториса (как она была доказана Виеторисом [23]) говорит, что если для всех $0 \leqslant r \leqslant m$ и всех $y \in \bar{Y}$, $H_{r}\left(f^{-1}(y)\right)=0$ (предполагаются гомологии Виеториса по mod два), то индуцированный гомоморфизм $f_{*}: H_{r}(X) \rightarrow H_{r}(Y)$ является изоморфизмом на для $r \leqslant m$ и на для $r=m+1$. Существуют примеры, показывающие, что аналогичная теорема для гомотопий неверна. Тем не менее, налагая некоторые локальные условия, Смейл [22] доказал аналогичную теорему для гомотопий (ниже приведена ее формулировка для компактных пространств). Пусть $f: X \rightarrow Y$ отображение связных пространств $X$ и $Y, Y=f(X)$, $X \in \mathrm{LO}^{m}$ и для всех $y \in Y, f^{-1}(y)$ является локально связным и связным в размерности $m-1$ пространством (т. е. $f^{-1}(y) \in \mathrm{LC}^{m-1}, \mathrm{C}^{m-1}$ ). Тогда $Y \in \mathrm{LC}^{m}$ и индуцированный гомоморфизм $f_{\#}: \pi_{r}(X) \rightarrow \pi_{r}(Y)$ является изоморфизмом на для всех $0 \leqslant r \leqslant m-1$ и на для $r=m$.

Основная цель данной работы состоит в доказательстве теоремы Виеториса в категории шейпов, в которой на пространства $X$ и $f^{-1}(y)$ не накладывается никаких локальных условий, но, как уже говорилось, в теореме Смейла просто зачеркнуть $\mathrm{LC}^{m}$ и $\mathrm{LC}^{m-1}$ нельзя, поэтому от множеств

(*) После представления статыи автор узнал, что некоторые аналогичные результаты получены для точечных отображений в [26], для клеточно-подобных отображенцй в [27], [28] и для $\vartheta \nabla^{n}$-отображений $\propto$ [25] 